\title{
Braking Indices for Twenty Pulsars
}

\author{
Simon Johnston ${ }^{1}$ and David Galloway ${ }^{1,2}$ \\ ${ }^{1}$ Research Centre for Theoretical Astrophysics, Univ. Sydney, Australia \\ ${ }^{2}$ School of Mathematics and Statistics, Univ. of Sydney, Australia
}

\begin{abstract}
We have derived a formula for the braking index of pulsars via integration rather than the conventional differentiation method. We applied the method to 20 pulsars and find that virtually all the derived braking indices are dominated by the effects of (unseen) glitches, the recovery from which corrupts the value of $\dot{\nu}$. However, any real, large, positive braking index has implications for magnetic field decay and offers support to recent models of pulsar evolution.
\end{abstract}

\section{The Method}

Neutron stars are powered by rotational kinetic energy and lose energy by accelerating particle winds and by emitting electromagnetic radiation at their rotation frequency, $\nu$. The rotation frequency thus decreases with time and this slowdown is usually described by the relation $\dot{\nu}=-K \nu^{n}$. Here, $K$ is a positive constant which depends on the moment of inertia and the magnetic dipole moment of the neutron star and $n$ is the braking index. Conventionally, the braking index is derived by differentiation yielding $n=\frac{\nu \ddot{\nu}}{\dot{\nu}^{2}}$. In a highly simplified model in which the spin-down torque arises from dipole radiation at the rotation frequency, one expects $n=3$.

Instead of differentiating equation the spin-down equation, we integrate from a time $t$ to $t+T$ to obtain

$$
n=1+\frac{\nu_{1} \dot{\nu}_{2}-\nu_{2} \dot{\nu}_{1}}{\dot{\nu}_{1} \dot{\nu}_{2} T}
$$

This allows the braking index to be computed without the need to measure $\ddot{\nu}$. The advantage of this method is that, in principle, $\nu$ and $\dot{\nu}$ can be measured over a short interval of time and then re-measured $20 \mathrm{yr}$ later without the need for a phase connected solution over the whole $20 \mathrm{yr}$ time span.

\section{The Results}

Using equation 1, it should be possible to obtain an error on the braking index as low as $\sim 0.2$ for pulsars of age $\sim 10^{5} \mathrm{yr}$ discovered more than $20 \mathrm{yr}$ ago (especially those with low timing noise). We select pulsars in the following way. We construct an 'expected' value of $\ddot{\nu}$ for every pulsar, assuming a braking index 
of 3 and select all those with $\ddot{\nu}>10^{-27} \mathrm{~Hz} / \mathrm{s}^{2}$. We reject all those pulsars which are known to have glitched in the past and/or have high timing noise. We then searched the literature for at least two timing solutions for the remaining pulsars and with a $\dot{\nu}$ of sufficient accuracy to allow the error on the computed braking index to be less than 20 . Twenty pulsars were found which fit the above criteria.

The results are given in tabular form in Johnston \& Galloway (1999). In brief, 14 of the 20 pulsars have significant values of braking index. The eight pulsars with the smallest error bars all have positive braking indices (PSRs $\mathrm{B} 0540+23, \mathrm{~B} 0611+22, \mathrm{~B} 0656+14, \mathrm{~B} 0740-28, \mathrm{~B} 1915+13, \mathrm{~B} 2002+31, \mathrm{~B} 2148+52$ and $B 2334+61$ ). The four pulsars with large negative braking indices are PSRs $\mathrm{B} 0136+57, \mathrm{~B} 1719-37, \mathrm{~B} 1742-30, \mathrm{~B} 2000+32$ and $\mathrm{B} 2255+58$.

\section{Discussion}

The presence of glitches in pulsars can lead to spurious values of the braking index and we surmise that (unseen) glitches are the main cause for $n \neq 3$. If we assume that glitches cause $\Delta \dot{v} / \dot{v}$ to be positive in all cases and that the value of $\dot{\nu}$ recovers to nearly its original value, then large negative braking indices can be caused by glitches between timing solutions and positive braking indices arise when the glitch occurs before the epoch of the first timing solution. Alpar \& Baykal (1994), in a statistical survey of the frequency of pulsar glitches, showed that the number of glitches, $g$, in a sample of pulsars is given by $g=$ $\left(\frac{\delta \nu}{\nu}\right)^{-1} \sum T \frac{\dot{\nu}}{\nu}$ where $T$ is the time interval over which the pulsar is monitored and $\delta \nu / \nu$ is $1.74 \times 10^{-4}$.

Using this equation, one would expect 2.8 glitches to have occurred in the sample between the two timing solutions and 6.4 glitches to have occurred in the 25 years before the first timing solution. This is broadly in line with our results of 4 negative braking indices and 8 positive.

It may be that in one or more cases the braking index is indeed significantly larger than 3. What are the implications of this? According to Blandford \& Romani (1988), it implies that the magnetic field is decaying on a timescale of roughly $50 \mathrm{kyr}$. This is a surprising result but may be in line with recent pulsar models such as that proposed by Chen, Ruderman \& Zhu (1998).

One is forced to conclude that the initial starting premise that the spin-down of a pulsar can be described as a simple power law in $\nu$ is highly questionable. In virtually all young pulsars, and in most older ones, complex glitch behaviour appears to be dominating the spin-down behaviour. This glitch behaviour, coupled with changes in the magnetic field strength over time, indicates that the entire concept of a 'braking index' must be treated with some caution.

\section{References}

Alpar M. A., Baykal A., 1994, MNRAS, 269, 849

Blandford R. D., Romani R. W., 1988, MNRAS, 234, 57P

Chen K., Ruderman M., Zhu T., 1998, ApJ, 493, 397

Johnston, S., Galloway, D., 1999, MNRAS, 306, L50 